\title{
Der Dresdner Verleger Lothar Dunsch (1948-2013) und seine Editionen zur Geschichte der Naturwissenschaften und Medizin
}

Volker Klimpel

In seinem einen Leben war Lothar Dunsch forschender Chemiker mit einer glänzenden akademischen Laufbahn und internationalem Ansehen. Er wurde am 14. Februar 1948 im sächsischen Großröhrsdorf geboren und ist in Pulsnitz aufgewachsen - der Stadt Ernst Rietschels (1804-1861) ${ }^{1}$-, studierte nach einer Maschinenbaulehre Chemie an der Bergakademie Freiberg (heute Technische Universität Bergakademie Freiberg) und promovierte dort 1973 mit dem Thema „Zur anodischen Oxydation des Anilins in gesättigter NaClLösung“ zum Dr. rer. nat. Von 1974 bis 1978 arbeitete Dunsch als wissenschaftlicher Assistent am Institut für Festkörper und Werkstoffforschung und bis 1991 als Arbeitsgruppenleiter des Instituts für Technologie der Polymere, beide in Dresden. Seine Arbeitsgebiete waren die Elektrochemie der Polymeren, die Spektroskopie und die Fullerene.

Nach einem Gastaufenthalt am HeyrovskyInstitut $^{2}$ in Prag leitete Dunsch seit 1992 die Abteilung „Elektrochemie und leitfähige Polymere" am Dresdner Leibniz-Institut für Festkörper und Werkstoffforschung (IWF). 1996 habilitierte er sich mit der Arbeit „ESR ${ }^{3}$ spektroskopische in situ Methode in der Elektrochemie“ an der Technischen Universität Dresden, wo er 2002 zum Honorarprofessor berufen wurde. 2009 gründete Professor Dunsch das „Zentrum für Spektroelektrochemie“ am IWF Dresden, im Dezember 2012 erhielt er von der Slowakischen Technischen Universität in Bratislava die Ehrendoktorwürde verliehen.

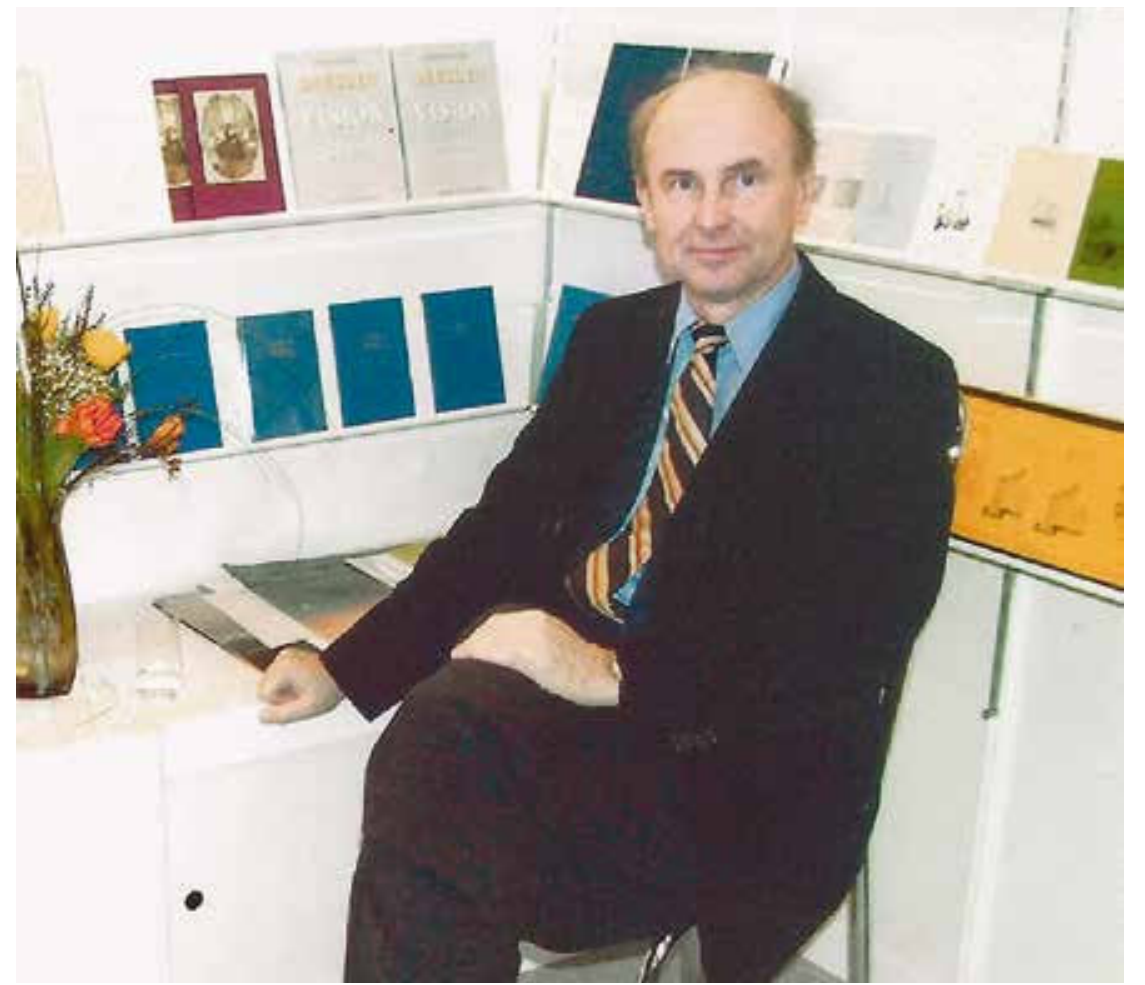

Soweit die Kurzbiographie des Wissenschaftlers Dunsch, den Vortragsreisen in die fernsten Länder führten und dessen Publikationsverzeichnis über 300 Stellen aufweist. ${ }^{4}$ Schon in den 1980er Jahren beschäftigte sich Dunsch auch mit der Geschichte seines Fachgebietes und ihren bedeutenden Persönlichkeiten. In der Teubnerschen ${ }^{5}$ Reihe „Biographien hervorragender Naturwissenschaftler, Techniker und Mediziner" veröffentlichte Dunsch 1982 das
Der Verleger Lothar Dunsch in seinem Stand auf der Leipziger Buchmesse, 2010.

Foto: Anette Dunsch 


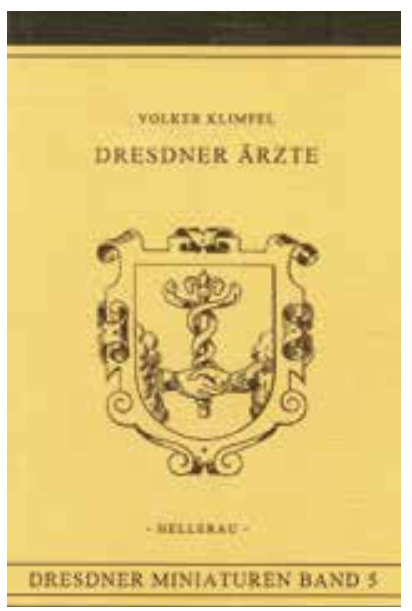

Volker Klimpel, Dresdner Ärzte.

1 Bildhauer, Schöpfer des Goethe-Schiller-Denkmals in Weimar. Über ihn schrieb Dunsch das Büchlein „Ernst Rietschel in Dresden“. Hellerau-Verlag 2004. Der Enkel Hans Rietschel (1878-1970) war Professor der Pädiatrie, sein Urenkel Ernst Theodor Rietschel (geb. 1941) ist Professor der Chemie.

2 Benannt nach Jaroslav Heyrovsky (1890-1967), tschechischer Physiko-Chemiker.

Elektronenspinresonanz.

4 http://www.ifw-dresden.de/de prof-lothar-dunsch [12. 03. 2014]

5 Wissenschaftlicher Verlag in Leipzig, gegründet von Benedictus Gotthelf Teubner (17841856), bestand von 1811 bis 2008.

6 Zum Beispiel Krafft, Fritz (Hrsg.): Lexikon großer $\mathrm{Na}-$ turwissenschaftler. Vorstoß ins Unbekannte. Wiesbaden 2003, S. 49,115 .

7 Dunsch, Lothar: Das Porträt: Wilhelm Ostwald (1853-1932). In: Chemie in unserer Zeit. Bd. 16. Weinheim 1982, S. 186-196.

8 Dunsch, Lothar: Geschichte der Elektrochemie. Ein Abriss. Leipzig 1985.

9 Hier befinden sich heute das Wilhelm-Ostwald-Archiv, ein Museum und eine Begegnungsstätte.

10 Szöllösi-Janze, Margit: Fritz Haber. München 1998; Daiber, Jürgen: Experimentalphysik des Geistes. Novalis und das romantische Experiment. Göttingen 2001; Hackenholz, Dirk: Die elektrochemischen Werke in Bitterfeld 1914-1945. Ein Standort der IG-Farbenindustrie AG. Münster 2004.
Porträt des englischen Chemikers Sir Humphry Davy (1778-1829) und 1986 das Lebensbild des schwedischen Chemikers Jöns Jacob von Berzelius (1779-1848). Beiden fühlte er sich durch seine Studien und speziellen Forschungen besonders verbunden. Und von beiden ist auch ihre Verbindung zur Medizin anzumerken. Berzelius hat regulär an der Universität von Uppsala Medizin studiert und promoviert, hat am Collegium medicum in Stockholm gearbeitet und ist dort Armenarzt gewesen, bevor er zur Chemie und Pharmazie „umschwenkte“, dies jedoch wieder an der Chirurgischen Schule von Stockholm. Davy kam schon im Alter von 16 Jahren zu einem Chirurgen und Apotheker in die Lehre, wo er sich die Grundlagen chemischen Arbeitens aneignete, die dann für seinen späteren Lebensweg bestimmend wurden. Während der eine mit der Bestimmung chemischer Elemente und ihrer Atomgewichte noch über 150 Jahre später unseren Dresdner Wissenschaftler beeindruckte, kam der andere Dunsch unter anderem mit der Elektrostatik und dem Kohlenbogenlicht nahe. Dunschs Arbeiten zu diesen Naturforschern wiederum waren von solcher Relevanz, dass sie in der Literatur einschlägiger Lexika aufgeführt wurden. ${ }^{6}$ In diesen auch wissenschaftshistorisch produktiven Jahren erschienen von Dunsch 1982 das Porträt Wilhelm Ostwalds (1853-1932), des Ionenforschers und Begründers der modernen Physikalischen Chemie ${ }^{7}$, sowie 1985 die dann mehrfach aufgelegte "Geschichte der Elektrochemie“. ${ }^{8}$ Mit Ostwald, dem Balten in Großbothen bei Leipzig', hat sich Dunsch mehrfach beschäftigt. Das Ergebnis waren außer der oben genannten die Schriften „Wilhelm Ostwald und die Naturwissenschaften“ (1978), „Forschen und Nutzen - Wilhelm Ostwald zur wissenschaftlichen Arbeit" (1978 ff., zusammen mit Günther Lotz und Uta Kring) und „Ein Fundament zum Gebäude der Wissenschaften - 100 Jahre ,Ostwalds Klassiker der exakten Wissenschaften (18891989)'. Zu seinem wohl bedeutendsten wissenschaftshistorischen Werk wurde Dunschs Abriss „Geschichte der Elektrochemie“, in der er den Weg dieser Disziplin von den Anfängen bis zu Luigi Galvani (1737-1798), Alessandro Volta (1745-1827) und Michael Faraday (1791-1867) nachzeichnet, dabei die Entwicklung der Stromquellen und Batterien beschreibt und zu Hermann Helmholtz (1821-1894) hinführt. Weitere Kapitel betreffen die Geschichte der Elektrolyte, der Elektrolyse und der Elektrodenkinetik. Spätere
Autoren sind in ihren Monografien immer wieder auf diese grundlegende Arbeit von Dunsch zurückgekommen. ${ }^{10}$

In seinem anderen Leben erfüllte sich Lothar Dunsch 1990 - die veränderten politischen Verhältnisse und die neu gewonnene Freiheit machten es möglich - einen langgehegten Traum: mit einem Kompagnon, der bald wieder ausstieg, gründete er den Hellerau-Verlag in Dresden. Die materielle Grundlage dafür lieferte ihm sein Hauptberuf. Mit dem Verlag in der Inneren Neustadt sah sich Dunsch in der Tradition des Hellerauer Verlegers Jakob Hegner (1882-1962), einem Pionier des „ästhetischen Buchdrucks“, der Paul Claudel (1868-1955), Else Lasker-Schüler (18691945), Rainer Maria Rilke (1875-1926) und viele andere zu seinen Autoren zählte. In der bewegten Umbruchzeit von 1990 hat sich Dunsch auch politisch als Stadtverordneter bei den „Freien Bürgern“ engagiert, sich aber nach einer Legislaturperiode enttäuscht abgewandt. Umso mehr galt nun alle seine Kraft, die ihm neben der Lehr- und Forschungstätigkeit blieb, seinem Verlag. Hegners Satz „Einen Verlag macht man nicht, man lebt ihn, opfert sich für ihn" wurde Lothar Dunschs Leitmotiv. Und einen weiteren Ausspruch von Hegner zitierte Dunsch gern: „Verleger müssen zäh sein wie Katzen“, was seinen ständigen Kampf ums Überleben als Ein-Mann-Verlag umschreibt. In seinen besten Zeiten brachte der Hellerau-Verlag jährlich fünf bis zehn Neuerscheinungen heraus, am Ende des Verlages waren circa 70 Titel im Programm. ${ }^{11}$ Viele davon beschworen den Genius loci von Hellerau und regten in ihrer Vielseitigkeit auch zur Beschäftigung mit der Lebensreformbewegung und der medizinischen Seite dieses Dresdner „Arkardien“ an, wie der Schriftsteller Peter de Mendelssohn (1908-1982) die Gartenstadt nannte, in der er aufgewachsen war. ${ }^{12}$ Von Mendelssohn verinnerlichte Dunsch als Verleger auch Folgendes:

Wer Bücher liebt, der liebt Menschen, wer Bücher sammelt, der sammelt Menschen, wer Bücher macht, der erschafft Menschen, und wer von Büchern erzählt, der erzählt, wenn er ehrlich und aufrichtig ist, von sich selbst“. ${ }^{13}$

In Hellerau hatten - auf der Basis der rhythmisch-musikalischen Kurse des Schweizer Komponisten und Musikpädagogen Émile Jaques-Dalcroze (1865-1950) - die Heileurythmie und die Musiktherapie unserer Zeit 
ihre Wurzeln. In Hellerau behandelten fortschrittliche Psychiater wie Ernst Jolowicz (1882-1958) nach Methoden der Suggestion und Tanztherapie vor allem konzentrationsund verhaltensgestörte Kinder, und es gab ein ärztlich geleitetes „Tagesstätte und HeimErziehungsinstitut für seelenpflegebedürftige Kinder". Soziale Vereine hatten hier ihren Sitz, prominente Ärzte wie Otto Neustätter (1870-1941), Direktor am Deutschen Hygiene-Museum in Dresden, und Rudolf Neubert (1898-1992), der spätere Ordinarius für Sozialhygiene in Jena, lebten und arbeiteten in Hellerau. ${ }^{14}$

Im Hellerau-Verlag erschienen die Reihen „Opera Selecta Humanoria“, „Kleine Sächsische Bibliothek“, „Kursächsische Wanderungen“, „Dresdner Miniaturen“ und „Yess“ (für junge Lyriker und Prosaisten, bis 1998). Trotz der unbarmherzigen Konkurrenz im Verlagswesen und auf dem Buchmarkt landete der Hellerau-Verlag auch einige Bestseller wie „Als ich ein kleiner Junge war" von Erich Kästner, „Jugenderinnerungen eines alten Mannes“ von Wilhelm von Kügelgen, „Sächsische Geschichte“ von Otto Kaemmel oder „Hellerau - Die Gartenstadt als Künstlerkolonie“ von Hans-Jürgen Sarfert. Groß angelegt war 1997 die Neuauflage von Gustav Nieritz' (1795-1876) „Selbstbiographie“, ergänzt von einem profunden Nachwort des Dresdner Germanisten Günter Jäckel (19262011).

Dunsch hat noch im Februar seines Todesjahres 2013 einen Vortrag über den Armenschullehrer und Volksschriftsteller Nieritz und sein Leben im 19. Jahrhundert im Kügelgenhaus zu Dresden gehalten. Ein weiterer Meilenstein war 2002 die Edition des historisch-biographischen Lexikons „Berühmte Dresdner" mit 995 Kurzbiographien. ${ }^{15}$ Dass Dunsch nicht nur ein Mann des Labors, der Formeln und des Hörsaals war, sondern auch die Feder durchaus meisterhaft zu handhaben verstand, hatte er mit seinen wissenschaftshistorischen Abhandlungen schon hinlänglich bewiesen. Als Herausgeber und Autor in seinem eigenen Verlag bekräftigte er einmal mehr, wie ihm das Wort zu Gebote stand, nachzulesen beispielsweise in seinem Nachwort zu Charles Darwins „Das Leben meines Großvaters Erasmus Darwin“ (2003) oder in der Neuübersetzung mit Kommentar und Nachwort von Johann Keplers „Vom sechseckigen Schnee“ (2005). Beides sind bibliophile Ausgabe aus der Reihe „Opera Selecta Humanoria“. Bei der Darwinschen Biographie machte sich Dunsch die Mühe, anhand der 2003 in Cambridge nach den originalen Druckfahnen erschienenen Ausgabe dank seiner vorzüglichen Englischkenntnisse eine neue Übersetzung vorzunehmen und $\mathrm{zu}$ kommentieren. Mit Darwin ist wiederum ein Bezug zur Medizin gegeben, denn schon der Großvater und der Vater waren Ärzte, und auch Charles Darwin hat zunächst in Edinburgh Medizin studiert, wenn auch nur kurz und ohne Begeisterung. Die biographische Skizze über „Ehrenfried Walther von Tschirnhaus" "16 von Rudolf Zaunick (1893$1967)^{17}$ hat Dunsch ebenso neu herausgegeben und kommentiert wie die „Lebenserinnerungen eines deutschen Malers" von Ludwig Richter (2001 und 2008). Als Herausgeber hat Dunsch dem Tschirnhaus-Band eine lesenswerte biographische Notiz zu Rudolph Zaunick beigegeben, in der er die Bedeutung des Hochschulpädagogen und Wissenschaftshistorikers herausarbeitet, der unter anderem auch die „Lebenserinnerungen und Denkwürdigkeiten" von Carl Gustav Carus (17891869) edierte, übrigens in dem Dresdner Verlag von Wolfgang Jess (1885-nach 1944 verschollen), dem sich Dunsch mit seiner YessBuchreihe verbunden sah. Auch Zaunicks Rolle als Direktor der „Leopoldina“ ${ }^{18}$ und Herausgeber der weltberühmten „Acta Historica Leopoldina" wird entsprechend gewürdigt. $^{19}$ In seinem von 1994 bis 2004 in 10 Bänden erschienenen Hellerau-Almanach, seinem „Herzens- und Schmerzenskind“, ist der Verleger ebenfalls oft mit eigenen kulturgeschichtlichen Miniaturen hervorgetreten, die sich durch einen gepflegten, ja poetischen Stil auszeichnen und so gar nicht an den nüchternen Naturwissenschaftler denken lassen.

Die Heilkunde hat Lothar Dunsch stets als Teil der sächsischen Kultur betrachtet und es als schmerzlich empfunden, dass erst in den letzten Jahren in einigen Periodika die Medizingeschichte Platz gefunden hat und seit Heinz Egon Kleine-Natrops „Das heilkundige Dresden“ von 1964 keine größere Übersicht $\mathrm{zu}$ diesem Thema vorgelegt worden ist. $^{20}$ So wurde er auch auf diesem Felde tätig, nachdem er bereits 1994 die artverwandte „Pharmaziegeschichte Dresdens“ von Heinz Glodschei herausgebracht hatte, eine weitere Auflage dieses Bandes 3 der „Dresdner Miniaturen“ erschien 1996. Darin werden zum ersten Mal die Entwicklung der Dresdner Apotheken, die pharmazeutischen Unternehmen und Lehranstalten Dresdens und ihre Protagonisten dargestellt. 1998 entschloss sich Dunsch, ein historisch-biographisches

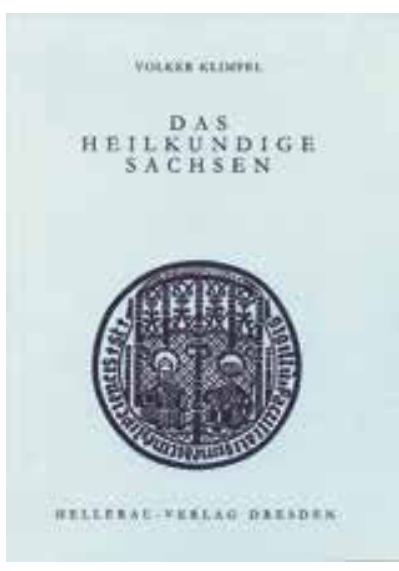

Volker Klimpel, Das heilkundige Sachsen.
11 Gärtner, Tomas: Der Sinn fürs Besondere. Lothar Dunsch, der Leiter des Hellerau-Verlages, ist im Alter von 65 Jahren gestorben. Dresdner Neueste Nachrichten vom 4. 12. 2013, S. 10.

12 Dunsch, Lothar: Arkardien und Hellerau. In: Hellerau Almanach 5. Hrsg. v. Lothar Dunsch. Dresden 1998, S. 59-72.

13 Zitiert nach Dunsch, Lothar: Jakob Hegner und sein Verlag in Hellerau. In: Dresdner Hefte 76 (2003), S.60.

14 Klimpel, Volker: Medizinisches aus Hellerau. Ärzteblatt Sachsen 10/2011, S. 546-549.

15 Klimpel, Volker: Berühmte Dresdner. Historisch-biographisches Handbuch bedeutender Persönlichkeiten, geboren in Dresden. Dresden 2002.

16 Naturforscher und Universalgelehrter der Aufklärungszeit, lebte von 1651 bis 1708, hat mit seiner „Medicina mentis“ (1687 ff.), seinen Brennspiegeln und Linsenapparaten die Naturwissenschaft und Medizin seiner Zeit beeinflusst

17 Pädagoge und Wissenschaftshistoriker, Professor in Dresden und Halle/Saale.

18 Deutsche Akademie der Naturforscher, 1652 in Schweinfurt gegründet, seit 1878 mit Sitz in Halle/Saale.

19 Dunsch, Lothar: Biographische Notiz zu Rudolph Zaunick vom Herausgeber. In: Rudolph Zaunick: Ehrenfried Walther von Tschirnhaus. Dresden 2001, S. 97-112. 
20 Medizinhistorische Beiträge finden sich in den vom Dresdner Stadtmuseum herausgegebenen "Dresdner Geschichtsbüchern“ ab 2007, im Themenheft „Gesundheitswesen in Dresden “ der Dresdner Hefte Bd. 113 (2013) und in der dreibändigen "Geschichte der Stadt Dresden“ (2005-2006)

21 Klimpel, Volker:Dresdner Ärzte. Historisch-biographisches Lexikon. Dresden 1998.

22 Vorgestellt am 12. März 2009 im Rahmen von „Leipzig liest“ auf der Leipziger Buchmesse und zum 20-jährigen Jubiläum des Hellerau-Verlages am 30. August 2010 im Deutschen Hygiene-Museum Dresden.

23 Pädagoge, Schriftsteller und Gymnasialdirektor in Wurzen.

24 Kunsthistoriker und Museumsdirektor in Dresden. Von ihm erschien in neuer Ausgabe 2002 im Hellerau-Verlag der „Führer durch das Grüne Gewölbe zu Dresden“.

25 Klimpel, Volker: Werner Forssmann in Dresden. Hellerau Almanach 10. Hrsg. v. Lothar Dunsch. Dresden 2004, S. 9-19.

26 www.hellerau-buergerverein.de/ index.php/aktuelles/news/114ende-des-hellerau-verlages

27 Siehe Gesamtkatalog 2011: ZweiJahrzehnteKulturgeschichte Hel-lerau-Verlag Dresden 2011
Lexikon „Dresdner Ärzte“21 als Band 5 der „Miniaturen“ herauszubringen, versehen mit einer medizinhistorischen Einführung und einer Zeittafel. Anhand von Leben und Werk der Ärzte wird auch zu den Anfängen von medizinischen Spezialdisziplinen wie $\mathrm{Au}$ genchirurgie, plastische Chirurgie, Homöopathie, Parasitologie, Endoskopie und Urologie in der Elbestadt hingeleitet. Der Nachfolgeband „Dresdner Ärzte des 20. Jahrhunderts“ liegt als Manuskript vor, konnte aber durch den am 28. November 2013 in Dresden erfolgten Tod von Lothar Dunsch nicht verwirklicht werden.

In diese Reihe fügte sich als Band 9 auch die "Astronomiegeschichte Dresdens" von Jürgen Helfricht ein (2001). Es sollten nach 1998 noch weitere Editionen mit medizinhistorischem Einschlag folgen, die einen nicht geringen verlegerischen Mut erforderten: "Das medizinische Dresden" (2009) ${ }^{22}$ und „Das heilkundige Sachsen" (2011), beide vom Verfasser des Ärzte-Lexikons und beide topografisch angelegt. Das Wagnis am Experiment entnahm der Verleger wohl seinem Chemikerberuf. „Das medizinische Dresden“ ist ein Führer durch die Medizingeschichte der Stadt in Form eines längeren Spaziergangs, auf dem die wichtigsten Orte und Ereignisse der Heilkunde dargestellt werden, illustriert von zahlreichen Abbildungen und verknüpft mit Geschichten am Rande des Geschehens. Immer wieder stößt der Leser auch auf enge Verbindungen zwischen Medizin und Kunst in Dresden. Im „Heilkundigen Sachsen“ wird die Medizin- und Krankenhausgeschichte Sachsens von den Anfängen bis zur Gegenwart abgehandelt und dazu die Form einer Reise von West nach Ost und von Nord nach Süd durch den Freistaat gewählt. Es finden sich viele Spezifika in der Entwicklung der Heilkunde in den Regionen und bedeutende Persönlichkeiten. Den Abschluss bilden medizinische Begriffe, die auf aus Sachsen stammende oder in Sachsen tätig gewesene Ärzte zurückzuführen sind und ihren Einfluss auf die gesamte Medizin belegen. Dunsch hatte zunächst ein auf die Medizin bezogener Text-Bild-Band in Art des „Bilder-Atlas zur Sächsischen Geschichte“ von Otto Eduard Schmidt (1856-1945) $)^{23}$ und Jean Louis Sponsel $(1858-1930)^{24}$ vorgeschwebt, was aber nicht auf die Gegenliebe des Autors stieß und schließlich zu der Reiseform der medizinhistorischen Beschreibung führte. In seinem zehnten und letzten Almanach gab Lothar Dunsch seinem medizinhistorischen Autor Gelegenheit, die Dresd- ner Episode des Chirurgen und Nobelpreisträgers Werner Forßmann (1904-1979) darzustellen. Forßmann hatte von 1935 bis 1938 als 1. Oberarzt bei Professor Albert Fromme (1881-1966) an der Chirurgischen Klinik am Krankenhaus Dresden-Friedrichstadt gearbeitet. Seine Selbstversuche mit dem Herzkatheter, die ihn berühmt machten, lagen da allerdings schon sechs Jahre zurück. ${ }^{25}$ Ein geplantes Buch mit dem Titel „Dresdner Exlibris“, verfasst von dem Medizinhistoriker und Spezialisten für Ärzte-Exlibris Albrecht Scholz (1940-2013) in Zusammenarbeit mit dem Historiker Konstantin Hermann, kam durch Scholz' und Dunschs Tod nicht zustande.

Lothar Dunsch hat von seinen Autoren nie einen Druckkostenzuschuss verlangt und in heute einzigartiger Weise immer allein das Risiko getragen. Er zählte zu den letzten Idealisten des Metiers und pflegte stets einen sehr persönlichen Kontakt zu seinen Autoren. Der Bürgerverein des Dresdner Stadtteils Hellerau, der dem Verlag seinen Namen gab, hat Lothar Dunsch einen einfühlsamen Nachruf gewidmet: „Mit dem Tod von Lothar Dunsch, seinem Gründer und Chef, stirbt auch der Hellerau-Verlag. Der Chemie-Professor starb im Herbst letzten Jahres nach schwerer Krankheit. 1990 gründete er aus Interesse an der sächsischen Kulturgeschichte seinen eigenen Verlag, quasi als Hobby, und sah sich in einer Tradition mit den frühen Hellerauer Verlegern, wie Jakob Hegner. Mit seiner Verlagsarbeit hat Lothar Dunsch maßgeblich zur Renaissance von Hellerau nach der Wende beigetragen. Viele Bücher über die Geschichte von Hellerau, die in rein kommerziellen Verlagen keine Chance gehabt hätten, sind von ihm veröffentlicht worden " ${ }^{26}$

Gleiches gilt für die sächsische Wissenschaftsgeschichte. Sie und insbesondere die Medizinhistoriographie haben in Lothar Dunsch einen stets aufgeschlossenen und aktiven Förderer ihrer Belange verloren. Was bleibt, ist die Literatur des Hellerau-Verlages. ${ }^{27}$

\section{Autor \\ Dr. Volker Klimpel \\ Dresden}

